\title{
LV-EV-1
}

\section{Laparoscopic S1 resection}

\author{
Yang-seok $\mathrm{KOH}^{*}$
}

Chonnam National University Hwasun Hospital, Hwasun, Korea

Lecture: Laparoscopic isolated caudate lobectomy remains a challenging procedure because of its deep location and proximity to the important vessels. Despite of the popularity of laparoscopic liver resection, laparoscopic isolated caudate lobectomy is not commonly performed because of the rarity of isolated caudate lesion in addition to its challenging nature of procedure. In this expert video session, the author would present two cases of isolated S1 (Spigelian lobe) resection for a colorectal liver metastasis and recurred hepatocellular carcinoma respectively. 